\title{
The Cosmological Mass Function in the Zel'dovich Approximation
}

\author{
Sergei F. Shandarin \\ University of Kansas, Lawrence, KS
}

\begin{abstract}
The Press-Schechter theory of the cosmological mass function and its modifications allow to constraint cosmological scenarios of the structure formation. Recently a few new models have been suggested that explored the influence of anisotropic collapse on the shape of the mass function. I discuss in more detail a particular model that assumes that a fluid particle becomes a part of a gravitationally bound halo when the smallest eigenvalue of the deformation tensor of the filtered initial density field reaches a certain threshold (like the filtered density contrast reaches the threshold in the Press-Schechter formalism). Choosing the smallest eigenvalue guarantees that the fluid particle in question experiences collapse along all three axes. The model shows a better agreement with the N-body simulations than the standard Press-Schechter model.
\end{abstract}

\section{Introduction}

The derivation of the distribution of masses in gravitationally bound objects is one of the principle goals of the theory of the structure formation (for a review see Monaco 1998). Comparing the theoretical mass function with observations provides important constraints on the cosmological models (see e.g. Bond \& Myers 1996, Bahcall \& Fan 1998, Reichart et al. 1999). Rich clusters of galaxies represent a particular interesting class of objects for two reasons. Firstly, they are the largest gravitationally bound objects in the universe and therefore represent rare events. As one of the consequences of being rare events clusters are particularly sensitive to some parameters of the cosmological models $\left(\Omega_{m}\right.$ and $\sigma_{8}$ ). Secondly, the formation of clusters is relatively simple process since it is primarily determined by the gravitational dynamics while other processes (hydro, thermal, etc) are less important than e.g. in the process of galaxy formation. As a result the numerical simulations of clusters of galaxies are more realistic and reliable than simulations of galaxy formation.

Measuring the mass function of galaxy clusters is not easy but recently a certain progress has been achieved for both optically (see e.g. Bahcall \& Cen 1993, Girardi et al. 1998) and X-ray (Reiprich \& Böhringer 1999) selected samples. Although there are systematic differences between mass functions obtained by different groups there is a general agreement in a broad sense.

Most of theoretical derivations of the cosmological mass function are based on the ideas of Press \& Schecter (1974) that can be summarized as follows: 
- The mass fraction $(F(>M))$ in gravitationally bound objects with masses greater than $M$ can be estimated as the fraction of mass satisfied the collapse condition at this scale $\left(\Psi\left(\delta_{M}>\delta_{c}\right)\right): F(>M)=2 \Psi\left(\delta_{M}>\delta_{c}\right)$.

- The collapse condition is local i.e. it can be expressed in terms of the quantities at one point.

- The quantity that determines the collapse is the linearly extrapolated filtered density contrast $\delta_{M} \geq \delta_{c}$ at a given point.

- The value of the threshold $\delta_{c}=3 / 20(12 \pi)^{2 / 3} \approx 1.69$ that corresponds to the collapse of a spherical top-hat model with the similar initial density contrast. It was assumed that the collapse of the spherical top-hat model approximately corresponded to the virialization of the gravitationally bound clump.

The mathematical aspects of the Press-Schechter formalism is outlined in the following section. Here I would like to discuss briefly some of ideas suggested since the formalism was proposed in 1974.

The excursion set approach (Peacock \& Heavens 1990, Bond et al. 1991) justified the assumption that $F(>M)=2 \Psi\left(\delta_{M}>\delta_{c}\right)$ in the case of a sharp $k$-space filter.

Many realized that the threshold $\delta_{c}=1.69$ does not provide the best fit to N-body simulations. Although some authors used the canonical value (e.g. Bond et al. 1991, Efstathiou et al. 1988) others preferred the lower values: $\delta_{c}=1.58$ (Bond \& Myers 1996), $\delta_{c}=1.44$ (Carlberg \& Couchman 1989), or even as low as $\delta_{c}=1.33$ (Efstathiou \& Rees 1988 and Klypin et al. 1995). Recently Shapiro et al. (1999) showed that the virialization of the top-hat model occurs when linear extrapolation of the density contrast reaches $\delta_{c} \approx 1.52$.

One of the major efforts in reduction of the discrepancy of the theory with simulations has been related to incorporating the anisotropic character of gravitational collapse. Bond \& Myers (1996) developed a model that incorporated both the Zel'dovich approximation on large scales and the collapse of a homogeneous ellipsoid on the nonlinear scale. Monaco (1995) suggested a different collapse condition that corresponded to the collapse along the first axis in the Zel'dovich approximation. Audit et al. (1997) incorporated some of the nonlinear effects into an anisotropic collapse model. Lee \& Shandarin (1998a) suggested to use the collapse condition corresponding to the collapse along all three axes as described by the extrapolation of the Zel'dovich approximation. Sheth \& Tormen (1999) obtained an analytic fit to the numerical mass function in the SCDM, OCDM and $\Lambda$ CDM models and then Sheth, Mo \& Tormen (1999)] provided a semianalytic derivation of the formula assuming an anisotropic collapse an incorporating some nonlocal effects. All but one models mentioned above assumed that the formation of a gravitationally bound object is related to the collapse along three axes. Only Monaco (1995)] assumed the collapse condition corresponding to the collapse along only the first axis.

In this talk I briefly review the Press-Schechter formalism. Then I describe the derivation of the mass function ( $\lambda_{3}$-function) in the Zel'dovich approximation. I compare the result with the standard Press-Schechter model and the model suggested by Sheth, Mo \& Tormen (1999), I briefly discuss the results of 
comparison of the $\lambda_{3}$-function with N-body simulations. Finally, I discuss the effect of the initial gravitational potential on the cosmological mass function and show that the clusters have a significant tendency to form in the troughs of the initial gravitational potential.

\section{The Press-Schechter Formalism}

The mass function $n(M)$ is the number density of gravitationally bound clumps with masses between $M$ and $M+d M$. Let $F(>M)$ be the fraction of the mass contained in the gravitationally bound objects with masses greater than $M$. Press and Schechter (1974) suggested the fraction $F(>M)$ and the mass function $n(M)$ can be related as

$$
n(M)=-\frac{\bar{\rho}}{M} \frac{\partial F}{\partial M}
$$

where $\bar{\rho}$ is the mean mass density in the universe and the minus sign reflects the fact that $F$ is a decreasing function of $M$.

Press and Schechter also made the assumption that the fraction of mass $F(>M)$ can be estimated as a fraction of mass $\Psi\left(\delta_{M}>\delta_{c}\right)$ in the initial density field filtered with the window function $W$ (corresponding to $\tilde{W}$ in $k$-space)

$$
\delta_{M}(\mathbf{x}, t)=D(t) \int \delta_{i n}\left(\mathbf{x}^{\prime}\right) W\left(\left|\mathbf{x}^{\prime}-\mathbf{x}\right| / R\right) d^{3} x^{\prime}
$$

satisfying the collapse condition $\delta_{M}>\delta_{c}$. Here $\delta=(\rho-\bar{\rho}) / \bar{\rho}$ is the density contrast, $D(t)$ is linear growth factor, $\mathbf{x}$ is the comoving coordinate. The mass $M$ and the linear scale $R$ of the filter are related as

$$
M=f_{W} \frac{4 \pi}{3} R^{3} \bar{\rho}
$$

where $f_{W}$ is a factor depending on the shape of the smoothing filter $W$. For a sharp k-space filter adopted here $f_{W}=9 \pi / 2 \approx 14.1$ and thus $M=6 \pi^{2} R^{3} \bar{\rho}$ (see e.g. Lacey \& Cole (1994)).

Assuming that the initial density contrast is Gaussian random field its pdf (probability distribution function) is

$$
p\left(\delta_{M}\right)=\frac{1}{\sqrt{2 \pi} \sigma_{M}} \exp \left[-\frac{\delta_{M}^{2}}{2 \sigma_{M}^{2}}\right],
$$

where the variance $\sigma_{M}^{2}$ is a function of mass $M$

$$
\sigma_{M}^{2}=\int \frac{d^{3} k}{(2 \pi)^{3}} P(k) \tilde{W}^{2}(k R),
$$

where $P(k)=\left|\delta_{k}\right|^{2}$ is the initial spectrum of perturbations and $\tilde{W}(k R)$ is the window function in the k-space.

Press and Schechter argued that a fluid element becomes a part of a gravitationally bound object of mass $M$ when its linearly extrapolated density contrast 
$\delta_{M}$ reaches the critical value $\delta_{c}=3 / 20(12 \pi)^{2 / 3} \simeq 1.69$. This corresponds to the collapse of the top-hat spherical perturbation having the initial density contrast similar to the fluid element in question. The collapse of a spherical top-hat model has been assumed approximately to correspond the virialization of the clump. Recently Shapiro et al. (1999) showed that the virialization corresponds to $\delta_{c} \simeq 1.52$ rather than to $\delta_{c} \simeq 1.69$.

The fraction of mass satisfying the collapse condition on scale $M$ is

$$
\begin{aligned}
\Psi\left(\delta_{M}>\delta_{c}\right) & =\frac{1}{\sqrt{2 \pi} \sigma(M)} \int_{\delta_{c}}^{\infty} \exp \left[-\frac{\delta_{M}^{2}}{2 \sigma^{2}(M)}\right] d \delta_{M} \\
& =\frac{1}{2} \operatorname{erfc}\left[\frac{\delta_{c}}{\sqrt{2} \sigma(M)}\right],
\end{aligned}
$$

where $\operatorname{erfc}(x)$ is the complementary error function. Assuming that $F(M) \approx$ $\Psi\left(\delta_{M}>\delta_{c}\right)$ one easily obtains the mass function $n(M)$ (eq. 1 $)$.

One obvious problem with this result is that the normalization integral

$$
\int_{0}^{\infty} d F \approx \Psi\left(\delta_{M=\infty}>\delta_{c}\right)=\frac{1}{2}
$$

meaning that only a half of the mass is contained in the gravitationally bound clumps. Press and Schechter renormalized $n(M)$ by introducing an additional factor of $2\left(F(M)=2 \Psi\left(\delta_{M}>\delta_{c}\right)\right.$

$$
\begin{aligned}
n_{p s}(M) & =-2 \frac{\bar{\rho}}{M} \frac{\partial \Psi}{\partial M}=2 \frac{\bar{\rho}}{M} \frac{d \sigma}{d M} \frac{\partial \Psi}{\partial \sigma} \\
& =-\sqrt{\frac{2}{\pi}} \frac{\bar{\rho}}{M} \frac{d \sigma}{d M} \frac{\delta_{c}}{\sigma^{2}(M)} \exp \left[-\frac{\delta_{c}^{2}}{2 \sigma_{M}^{2}}\right] .
\end{aligned}
$$

Later, the normalization problem was correctly resolved in the frame of the excursion set model (Peacock \& Heavens 1990 and Bond et al. 1991). The derivation of Press and Schechter did not take into account the so called cloudin-cloud problem. Function $\Psi\left(\delta_{M}>\delta_{c}\right)$ in eq.6 gives the fraction of mass that satisfies the collapse condition at the filtering scale $M$. However, some of the fluid particles may satisfy the collapse condition at larger filtering scales. In the correct model the fluid elements must be assigned to the clumps of mass $M_{1}$ being equal to the largest filtering mass at which the collapse condition is fulfilled. In the excursion set formalism this corresponds to the first crossing of the collapse threshold $\delta_{c}$ while $\delta$ evolves with the growth of $\sigma$.

An elegant method to normalize the mass function was suggested by Jedamzik (1995) (see also the discussion in Yano et al. (1996)) who derived the integral equation

$$
\Psi\left(\delta_{M}>\delta_{c}\right)=\int_{M}^{\infty} d M_{1} n\left(M_{1}\right) \frac{M_{1}}{\bar{\rho}} P\left(M, M_{1}\right) .
$$

that relates the fraction of the fluid elements firstly crossed the collapse threshold at the filtering scale $M_{1}\left(d M_{1} n\left(M_{1}\right) M_{1} / \bar{\rho}\right)$ and the fraction of mass satisfying the collapse condition at filtering scale $M\left(\Psi\left(\delta_{M}>\delta_{c}\right)\right)$. Function $P\left(M, M_{1}\right)$ is the probability that a fluid particle firstly crossed the collapse threshold at 
the scale $M_{1}$ satisfies the collapse condition at the scale $M$. In the case of the sharp k-space filter and Gaussian $\delta_{M}$ this probability is exactly equal to $1 / 2$ for all $M_{1}>M$. Thus, the integral equation (9) can be immediately solved for the mass function $n(M)$. The solution is the correctly normalized mass function of eq. 8 .

\section{Mass Function in the Zel'dovich Approximation}

The simplest theory describing the anisotropic character of the gravitational collapse in a generic case of random initial condition is the Zel'dovich approximation (Zel'dovich 1970, see also Shandarin \& Zel'dovich 1989 for a discussion). In particular, the Zel'dovich approximation provides a formula for an anisotropic collapse of a fluid element

$$
\rho(\mathbf{q}, t)=\frac{\bar{\rho}}{\left[1-D(t) \lambda_{1}(\mathbf{q})\right]\left[1-D(t) \lambda_{2}(\mathbf{q})\right]\left[1-D(t) \lambda_{3}(\mathbf{q})\right]},
$$

where $D(t)$ is the linear growth function and $\lambda_{1}(\mathbf{q}), \lambda_{2}(\mathbf{q})$ and $\lambda_{3}(\mathbf{q})$ are the eigenvalues of the initial deformation tensor. Using the ordering convention $\lambda_{1}(\mathbf{q})>\lambda_{2}(\mathbf{q})$ and $\lambda_{2}(\mathbf{q})>\lambda_{3}(\mathbf{q})$ the condition $1-D(t) \lambda_{1}(\mathbf{q})=0$ has been interpreted as a collapse of a fluid particle along one principle axis Zel'dovich 1970). Similarly the conditions $1-D(t) \lambda_{i}(\mathbf{q})=0(i=2,3)$ can be interpreted as collapses along the second and third principle axes.

Shandarin \& Klypin (1984) showed that the formation of gravitationally bound clumps was the best correlated with the maxima of the smallest eigenvalue $\left(\lambda_{3}\right)$ of the initial deformation tensor. Although the formation of the clumps may be also related to other pointlike singularities (Arnol'd et al. 1982) here we assume that a fluid particle becomes a part of a gravitationally bound clump of mass $M$ when its smallest eigenvalue $\lambda_{3}$ reaches the critical value $\lambda_{c}$ at the largest filtering scale $M$ (Lee \& Shandarin 1998a). The Zel'dovich approximation (eq.10) predicts that the collapse condition is $\lambda_{c}=1$ (it is assumed that $D(t)$ normalized to $D\left(t_{0}\right)=1$, where $t_{0}$ is the present time). However, because of multistreaming effect all fluid particles (except the set of measure zero) enter the multi-stream flow regions before they collapse. We approximately incorporate this complex effect by reducing the threshold $\lambda_{c}$ to a smaller value. The comparison with the Press-Schechter mass function as well as with the numerical mass function suggests that $\lambda_{c}=0.37$ is a reasonable choice.

The derivation of the mass function in the Zel'dovich approximation is similar to the Press-Schechter derivation except that the collapse condition is $\lambda_{3}(M)=\lambda_{c}$ instead of $\delta_{M}=\delta_{c}$. Doroshkevich (1970) derived the joint pdf of three eigenvalues

$$
p\left(\lambda_{1}, \lambda_{2}, \lambda_{3}\right)=\frac{3375}{8 \sqrt{5} \pi \sigma^{6}} \exp \left(-\frac{3 I_{1}}{\sigma^{2}}+\frac{15 I_{2}}{2 \sigma^{2}}\right)\left(\lambda_{1}-\lambda_{2}\right)\left(\lambda_{2}-\lambda_{3}\right)\left(\lambda_{1}-\lambda_{3}\right),
$$

where $I_{1}=\lambda_{1}+\lambda_{2}+\lambda_{3}, I_{2}=\lambda_{1} \lambda_{2}+\lambda_{2} \lambda_{3}+\lambda_{3} \lambda_{1}$ and $\sigma^{2}$ is the density contrast variance as defined in eq. 5. Integrating $p\left(\lambda_{1}, \lambda_{2}, \lambda_{3}\right)$ over two eigenvalues one can obtain the pdf of one of the eigenvalues. We are interested in the collapse 
along the third axis and therefore $p\left(\lambda_{3}\right)$ is of primary interest

$$
\begin{aligned}
p\left(\lambda_{3}\right) & =\frac{\sqrt{5}}{12 \pi \sigma}\left\{3 \sqrt{3 \pi} \exp \left(-\frac{15 \lambda_{3}^{2}}{4 \sigma^{2}}\right) \operatorname{erfc}\left(\frac{\sqrt{3} \lambda_{3}}{2 \sigma}\right)\right. \\
& +\sqrt{2 \pi}\left(20 \frac{\lambda_{3}^{2}}{\sigma^{2}}-1\right) \exp \left(-\frac{5 \lambda_{3}^{2}}{2 \sigma^{2}}\right) \operatorname{erfc}\left(\sqrt{2} \frac{\lambda_{3}}{\sigma}\right) \\
& \left.-20 \frac{\lambda_{3}}{\sigma} \exp \left(-\frac{9 \lambda_{3}^{2}}{2 \sigma^{2}}\right)\right\} .
\end{aligned}
$$

Repeating the derivation of the previous section using the pdf of eq.12 instead of eq. T- one arrives to an analog of the normalization integral equation (eq.9)

$$
\Psi\left(\lambda_{3}(M)>\lambda_{c}\right)=\int_{M}^{\infty} d M_{1} n\left(M_{1}\right) \frac{M_{1}}{\bar{\rho}} P\left(M, M_{1}\right),
$$

here $\Psi\left(\lambda_{3}(M)>\lambda_{c}\right)$ is the fraction of mass where $\lambda_{3}(M)>\lambda_{c}$ on the filter scale $M$.

Solving exactly eq. 13 is much more difficult than eq.9 because now $P\left(M, M_{1}\right)$ is not a constant. In the limit $M_{1}-M \ll M$ the probability $P=0.5$ as in eq.9 but in the limit $M_{1} \gg M$ it drops to $P=0.08$. Lee \& Shandarin (1998a) used the limiting value $P=0.08$ and analytically derived the mass function in the Zel'dovich approximation

$$
\begin{aligned}
& n(M)=-\frac{25 \sqrt{5}}{24 \pi} \frac{\bar{\rho}}{M} \frac{d \sigma}{d M} \frac{\lambda_{3 c}}{\sigma_{M}^{2}} \\
& \left\{3 \sqrt{3 \pi} \exp \left(-\frac{15 \lambda_{3}^{2}}{4 \sigma^{2}}\right) \operatorname{erfc}\left(\frac{\sqrt{3} \lambda_{3}}{2 \sigma}\right)\right. \\
& +\sqrt{2 \pi}\left(20 \frac{\lambda_{3}^{2}}{\sigma^{2}}-1\right) \exp \left(-\frac{5 \lambda_{3}^{2}}{2 \sigma^{2}}\right) \operatorname{erfc}\left(\sqrt{2} \frac{\lambda_{3}}{\sigma}\right) \\
& \left.-20 \frac{\lambda_{3}}{\sigma} \exp \left(-\frac{9 \lambda_{3}^{2}}{2 \sigma^{2}}\right)\right\} \text {. }
\end{aligned}
$$

In the following sections I compare the obtained result with the PressSchechter and Sheth-Mo-Tormen mass functions as well as with numerical simulations.

\section{Comparison of Three Analytic Mass Functions}

Both the Press-Schechter and $\lambda_{3}$-mass functions have a common factor $\frac{\bar{\rho}}{M} \frac{d \sigma}{d M}$ which depends on the initial spectrum and $f(\sigma) \equiv \partial F / \partial \sigma$ that completely characterizes a model. Thus, comparing different models is convenient by comparing $f(\sigma)$ as functions of $\sigma$. The Press-Schechter and $\lambda_{3}$-functions are

$$
f_{P S}(\sigma)=\sqrt{\frac{2}{\pi}} \frac{\delta_{c}}{\sigma^{2}} \exp \left(-\frac{\delta_{c}^{2}}{2 \sigma^{2}}\right)
$$




$$
\begin{aligned}
f_{\lambda_{3}}(\sigma) & =\frac{25 \sqrt{5}}{24 \pi} \frac{\lambda_{3 c}}{\sigma^{2}}\left\{3 \sqrt{3 \pi} \exp \left(-\frac{15 \lambda_{3}^{2}}{4 \sigma^{2}}\right) \operatorname{erfc}\left(\frac{\sqrt{3} \lambda_{3}}{2 \sigma}\right)\right. \\
& +\sqrt{2 \pi}\left(20 \frac{\lambda_{3}^{2}}{\sigma^{2}}-1\right) \exp \left(-\frac{5 \lambda_{3}^{2}}{2 \sigma^{2}}\right) \operatorname{erfc}\left(\sqrt{2} \frac{\lambda_{3}}{\sigma}\right) \\
& \left.-20 \frac{\lambda_{3}}{\sigma} \exp \left(-\frac{9 \lambda_{3}^{2}}{2 \sigma^{2}}\right)\right\} .
\end{aligned}
$$

Sheth and Tormen (1999) and Sheth, Mo and Tormen (1999) derived a new mass function that fits better the results of the N-body simulations

$$
f_{S M T}(\sigma)=A\left[1+\left(\frac{a \delta_{c}^{2}}{\sigma^{2}}\right)^{-q}\right] \sqrt{\frac{2}{\pi}} \frac{\sqrt{a} \delta_{c}}{\sigma^{2}} \exp \left(-\frac{a \delta_{c}^{2}}{\sigma^{2}}\right),
$$

here $a=0.707, q=0.3$ and the constant $A=0.322$ found from the normalization condition

$$
\int_{0}^{\infty} f(\sigma) d \sigma=1
$$

Choosing $a=1, q=0$ and the constant $A=1$ one obtains the Press-Schechter function. These three functions are shown in Fig. 1a. The small box shows the range of $\sigma$ where the theoretical mass functions were checked against N-body simulations by Sheth \& Tormen (1999) and Lee \& Shandarin (1999). Fig. 1b shows the ratios $f_{P S} / f_{S M T}$ and $f_{\lambda_{3}} / f_{S M T}$.

\section{Comparison with N-body Simulations}

Figure 2 shows the comparison of the $\lambda_{3}$-function with the numerical mass functions for the scale invariant initial spectra: $P(k) \propto k^{n}$ with $n=-1$ and $n=0$ (see for the details Tormen (1998)). The top panel $(n=-1)$ shows a quite good agreement of the $\bar{\lambda}_{3}$-function with the numerical mass function, while in the $n=0$ case the agreement is significantly worse. Fig. 3 shows the comparison of the $\lambda_{3}$-function with the N-body simulation of the SCDM model (Governato et al. (1999)]. At four epochs $(z=1.86,1.14,0.43$, and 0$)$ the $\lambda_{3}$-function is in a better agreement that the Press-Schechter mass function. A similar result has been reported by Sheth \& Tormen (1999).

\section{Large-Scale Biasing}

It has been noticed for sometime that the initial gravitational potential may noticeably affect the large scale structure. Kofman and Shandarin (1988) showed that the adhesion approximation predicts that the formation of voids is associated with positive peaks of the primordial gravitational potential. Sahni et al. (1994) studied the effect and measured a significant correlation between the sizes of voids and the value of primordial gravitational potential in numerical simulations of the adhesion model. Recently, Madsen et al. (1998) have demonstrated by N-body simulations that the underdense and the overdense regions are closely linked to the regions with the positive and the negative gravitational potential 
respectively. Lee \& Shandarin (1998b) showed that the initial potential also affects the masses of clusters.

In order to incorporate the primordial gravitational potential fluctuations term into the derivation of the mass function, we first derive the conditional probability density distribution $p\left(\delta \mid \varphi<-\varphi_{c}\right)\left(\varphi_{c}>0\right)$ :

$$
\begin{aligned}
p\left(\delta \mid \varphi<-\varphi_{c}\right)= & \frac{1}{\sqrt{2 \pi} \sigma_{\delta}} \exp \left(-\frac{\delta^{2}}{2 \sigma_{\delta}^{2}}\right)\left[1-\operatorname{erf}\left(\frac{\varphi_{c}}{\sqrt{2} \sigma_{\varphi}}\right)\right]^{-1} \times \\
& {\left[1+\operatorname{erf}\left(\frac{\kappa \frac{\delta}{\sigma_{\delta}}-\frac{\varphi_{c}}{\sigma_{\varphi}}}{\sqrt{2\left(1-\kappa^{2}\right)}}\right)\right] . }
\end{aligned}
$$

Here $\sigma_{\delta}^{2}, \sigma_{v}^{2}$, and $\sigma_{\varphi}^{2}$ are the density, velocity and the potential variances respectively; $\kappa=\langle\delta \cdot \varphi\rangle / \sigma_{\delta} \sigma_{\varphi}=\sigma_{v}^{2} / \sigma_{\delta} \sigma_{\varphi}$ is the crosscorrelation coefficient of the the density contrast $\delta$ smoothed on the scale $k_{c}$ and the primordial (unsmoothed) potential fluctuations $\varphi$. As a result, eq.11 for the conditional mass function $n\left(M \mid \varphi<-\varphi_{c}\right)$ becomes

$$
n\left(M \mid \varphi<-\varphi_{c}\right)=-\frac{\bar{\rho}}{M}\left(\frac{\partial F}{\partial \sigma_{\delta}} \frac{d \sigma_{\delta}}{d M}+\frac{\partial F}{\partial \sigma_{v}} \frac{d \sigma_{v}}{d M}\right) .
$$

The further calculation needs to be done numerically. Fig. 4 illustrates how the mass function depends on the initial potential in the CDM model with $\Gamma=\Omega h=0.25$ normalized to $\sigma_{8}=1$. The top panel shows the mass function for regions of positive and negative initial potential as well as unconditional mass function. The bottom panel show the ratio of conditional mass functions to unconditional one.

We also calculate the probability that a clump with mass $M$ is located in the potential regions satisfying the chosen condition, for instance, $\varphi<-\varphi_{c}$

$$
P\left(\varphi<-\varphi_{c} \mid M\right)=\frac{n\left(M \mid \varphi<-\varphi_{c}\right)}{n(M)} P\left(\varphi<-\varphi_{c}\right)
$$

where $P\left(\varphi<-\varphi_{c}\right)$ is the fraction of space satisfying the given condition (see Fig. 5).

The scale of the initial potential

$$
R_{\varphi}=\sqrt{3} \sigma_{\varphi} / \sigma_{\varphi^{\prime}}=\sqrt{3 \frac{\int_{k_{l}}^{\infty} d k k^{-2} P(k)}{\int_{0}^{\infty} d k P(k)}} \approx 120 h^{-1} \mathrm{Mpc}
$$

does not depend on any ad hoc scale; the dependence on $k_{l}$ is extremely weak $\left(\propto \sqrt{\ln \left(1 / k_{l}\right)}\right.$ for the Harrison-Zel'dovich spectra assumed here). The geometry of the gravitational potential does not evolve much on large scales Kofman \& Shandarin (1988), Madsen et al. (1998)). Therefore, the potential at present is very similar to the primordial one on scales much greater than the scale of nonlinearity. A simple explanation to this in the frame of the standard scenario of the structure formation is due to the fact that the mass has been displaced by the distance about $10 h^{-1} \mathrm{Mpc}$ (Shandarin 1993). Therefore, the potential on scales greater than, say, $30 h^{-1} \mathrm{Mpc}$ has been changed very little. 
For the model in question the scale of the primordial potential is found to be $R_{\varphi} \approx 120 h^{-1} \mathrm{Mpc}$. The scale of the density contrast field reaches this value $R_{\delta}=\sqrt{3} \sigma_{\delta} / \sigma_{\delta^{\prime}} \approx 120 h^{-1} \mathrm{Mpc}$ only after it is smoothed on $k_{c} \approx 0.017 \mathrm{hMpc}^{-1}$. The corresponding density variance on this scale is $\sigma_{\delta}\left(0.017 h \mathrm{Mpc}^{-1}\right) \approx 0.03$. On the other hand, the number of clumps with masses $10^{14}-10^{15} h^{-1} M_{\odot}$ can easily be $30 \%$ greater in the troughs of the potential than the mean density $n(>M)=0.5[n(>M \mid \varphi<0)+n(>M \mid \varphi>0)]$ (see Fig. 4). Thus, the bias factor $b$ (defined by the relation $\Delta n_{c l} / n_{c l}=b \Delta \rho_{m} / \rho_{m}$ ) reaches at least 10 on the scale about $120 h^{-1} \mathrm{Mpc}$.

Figure 5 demonstrates that the most massive clusters $\left(M>10^{15} h^{-1} M_{\odot}\right)$ are almost certainly located in the the troughs in the initial potential. The bias defined as the density contrast of the clusters with respect to the mass density contrast $b=\delta_{c l} / \delta \rho$ reaches the value $3-10$ on the scale of the potential $R_{\phi} \approx 120 h^{-1} M p c$ (Lee \& Shandarin 1998b).

\section{Summary}

In the talk I discussed new modifications of the Press-Schechter theory of the cosmological mass function. One assumes a different collapse condition that implies that a fluid particle becomes a part of a gravitationally bound object after it experiences collapses along three axes. The comparison with other models (Fig. 1) shows that it predicts about $25 \%$ more gravitationally bound clumps than the Sheth-Mo-Tormen model in the range $0.45 \geq \sigma \geq 3.1$ where the comparison with the N-body simulations has been done. A direct comparison with the Nbody simulations (Fig. 2 and 3) shows a quite good agreement although not as good as the Sheth-Mo-Tormen model. The $\lambda_{3}$-function based on the Zel'dovich approximation has been obtained analytically similarly to the Press-Schechter function. A drawback of the derivation is a quite crude approximation of the probability function $P\left(M, M_{1}\right)$ in the normalization integral eq.13. A more accurate normalization will be reported separately. The Sheth-Mo-Tormen model also suffer from a normalization problem: the shape of the mass function has been derived but the normalization has been enforced by demanding equality of eq. 17

Another modification is the conditional mass function showing that the clusters of galaxies tend to form in the troughs of the initial gravitational potential and avoid the peaks of the potential. The gravitational potential field

has a typical scale of about $120 h^{-1} M p c$ and as a result has an advantage of being independent of the arbitrariness of the smoothing scale (if the filter scale is smaller than roughly $50 h^{-1} M p c$ ) and at present it has almost same geometry as at the epoch of decoupling. Figures 4 and 5 quantify this large-scale biasing.

\section{Acknowledgments}

I am grateful to Ravi Sheth for useful discussions during the workshop. This work has been partly supported by the University of Kansas GRF 2001 grant. 


\section{References}

Arnol'd, V.I., S.F. Shandarin, \& Ya.B. Zeldovich 1982, Geophys. Astrophys. Fluid Dynamics, 20, 111

Audit, E., R. Teyssier, \& J-M. Alimi 1997, A\&A, 325, 439

Bahcall, N.A. \& R. Cen, 1993, ApJ, 407, L49

Bahcall, N.A. \& X. Fan 1998, ApJ, 504, 1

Bond, J.R., S. Cole, G. Efstathiou, \& N. Kaiser 1991, ApJ, 379, 440

Bond, J.R. \& S.T. Myers 1996, ApJS, 103, 1

Carlberg, R.G. \& H.M.P. Couchman 1989, ApJ, 340, 47

Doroshkevich, A.G. 1970, Astrofizika, 6, 581

Efstathiou, G. \& M.J. Rees 1988, MNRAS, 230, P5

Efstathiou, G., C.S. Frenk, S.D.M. White, \& M. Davis 1988, MNRAS, 235, 715

Girardi, M., S. Borgani, R. Giuricin, F. Mardirossian, \& M. Mezzetti 1998, ApJ, 506,45

Governato, F., A. Babul, T. Quinn, P. Tozzi, C.M. Bauch, N. Katz, \& G. Lake 1999, MNRAS, 307, 949

Jedamzik, K. 1995, ApJ, 448, 1

Klypin, A., S. Borgani, J. Holtzman, \& J. Primack 1995, ApJ, 444, 1

Kofman, L. A., \& S.F. Shandarin 1988, Nature, 334, 129

Lacey, C., \& S. Cole 1994, MNRAS, 271, 676

Lee, J., \& S.F. Shandarin 1998a, ApJ, 500,14

Lee, J., \& S.F. Shandarin 1998b, ApJ, 505, L75

Lee, J., \& S.F. Shandarin 1999, ApJ, 517, L5

Madsen, S., A.G. Doroshkevich, S. Gottlober, \& V. Muller 1998, A\&A, 329, 1

Monaco, P. 1995, ApJ, 447,23

Monaco, P. 1998, Fundamentals of Cosmic Physics, 19, 157

Peacock, J. A., \& A.F. Heavens 1990, MNRAS, 243, 133

Press, W. H., \& P. Schechter 1974, ApJ, 187, 425

Reiprich, T. H. \& H. Böhringer 1999, astro-ph/9908357 
Reichart, D.E., R.C. Nichol, F.J. Castander, D.J.Burke, A.K. Romer, B.P. Holden, C.A. Collins, \& M.P. Ulmer 1999, ApJ, 518,521

Sahni, V., B.S. Sathyaprakash, \& S.F. Shandarin 1994, ApJ, 431, 20

Shandarin, S.F. 1993, in Cosmic Velocity Fields, eds. F.R. Bouchet and M. Lachieze-Rey (Paris : Editions Frontieres), p. 383

Shandarin, S. F. \& Ya.B. Zel'dovich 1989, Rev. Mod. Phys., 61, 185

Shandarin, S. F. \& A.A. Klypin 1984, Sov. Astron., 28, 491

Shapiro, P.R., I.T. Iliev, \& A.C. Raga 1999, MNRAS, 307, 203

Sheth, R.K. \& G. Tormen 1999, MNRAS,1999, 308, 119

Sheth, R.K., H.J. Mo, \& G. Tormen 1999, astro-ph/9907024

Tormen, G., MNRAS, 297, 648

Yano, T., M. Nagashima, \& N. Gouda 1996, ApJ, 466, 1

Zel'dovich, Ya. B. 1970, A\&A, 5, 84 


\section{Figure Captions}

Fig. 1. (a) The fraction of mass $f=d F / d \sigma$ in the gravitationally bound objects as a function of $\sigma$ as predicted by the Press-Schechter model (short dashed line), Sheth-Mo-Tormen model (solid line), and $\lambda_{3}$-model (long dashed line). The small box shows the range of $\sigma$ where the models have been checked against N-body simulations (see Fig. 2 in Sheth, Mo \& Tormen (1999))

(b) The logarithm of the ratios $f_{P S} / f_{S M T}$ (short-dashed line) and $f_{\lambda_{3}} / f_{S M T}$ (long-dashed line).

Fig. 2. The square dots represent the numerical mass function with poissonian error bars. The solid line is the $\lambda_{3}$-mass function with $\lambda_{3 c}=0.37$ while the dashed, the dotted lines are the PS mass functions with $\delta_{c}=1.69,1.5$ respectively. The upper and the lower panels correspond to the $n=-1$ and the $n=0$ power-law models respectively. See also the top left panel of Fig.2 in Tormen (1998).

Fig. 3. The square dots represent the numerical data for the case of SCDM model with $\Omega=1, h=0.5$. The solid line is our mass function with $\lambda_{3 c}=0.37$, and the dashed, the dotted lines are the PS mass functions with $\delta_{c}=1.69,1.5$ respectively.

Fig. 4. In the upper panel the conditional cumulative mass function satisfying chosen potential condition is plotted. The solid, the long dashed, the dot-dashed, and the dashed lines correspond to the conditions $\varphi<-\sigma_{\varphi}, \varphi<0$, $\varphi>0$, and $\varphi>\sigma_{\varphi}$ respectively, while the dotted line represents the unconditional cumulative PS mass function. The shaded area is $1 \sigma$ fit to the observational cumulative mass function of rich clusters by Bahcall and Cen (1993). In the lower panel the ratio of the conditional cumulative mass functions to the unconditional one is plotted for each condition. The CDM spectrum with $\Gamma=0.25$ normalized to $\sigma_{8}=1$ has been used.

Fig. 5. The probability that a clump with mass $M$ can be found in the regions satisfying chosen potential condition is plotted. The heavy solid, the heavy dashed, the solid, the dashed, the long dashed, and the dot-dashed lines correspond to the condition $\varphi<0, \varphi>0, \varphi<-\sigma_{\varphi},-\sigma_{\varphi}<\varphi<0,0<\varphi<\sigma_{\varphi}$, and $\varphi>\sigma_{\varphi}$ respectively. 

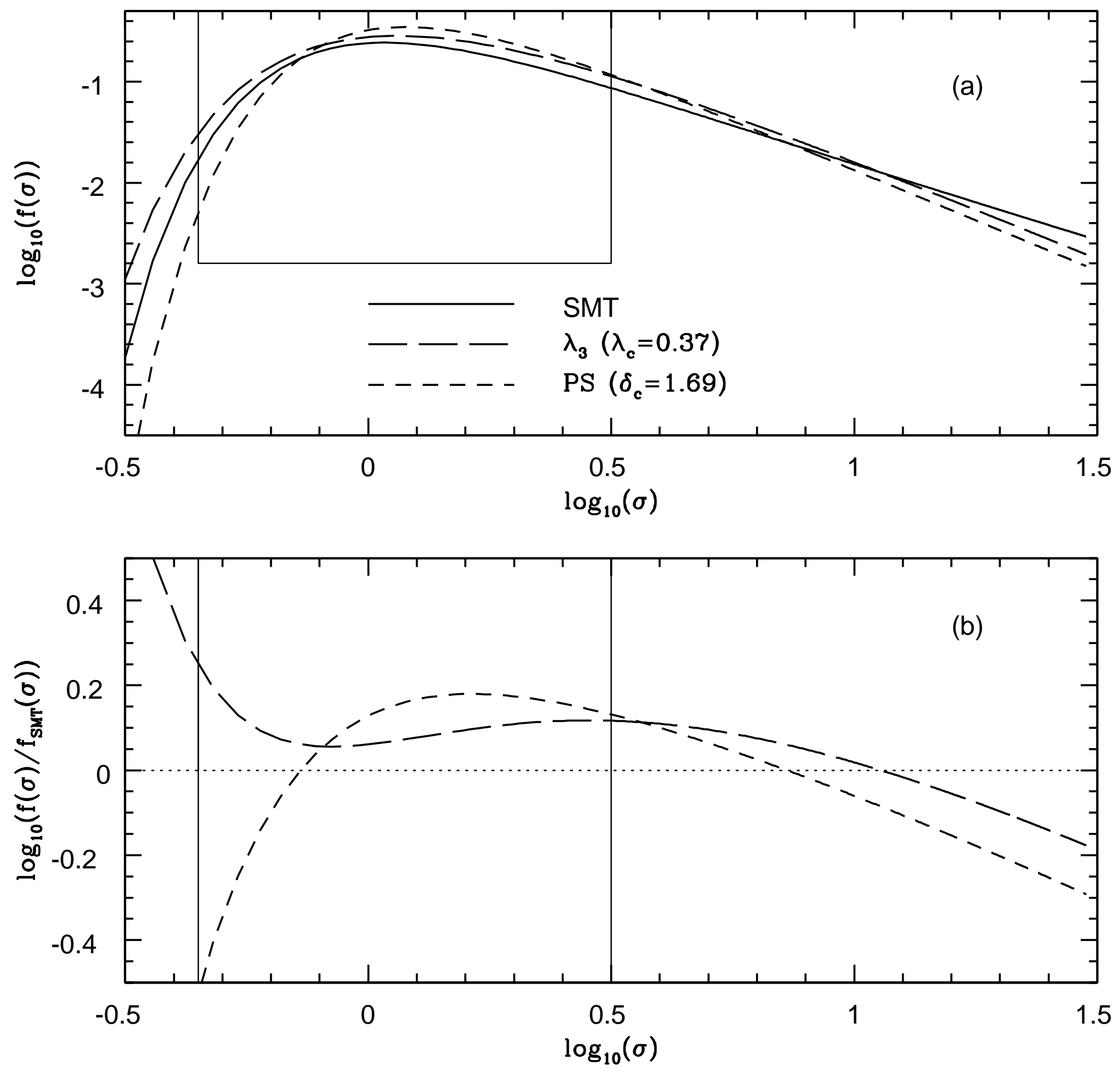

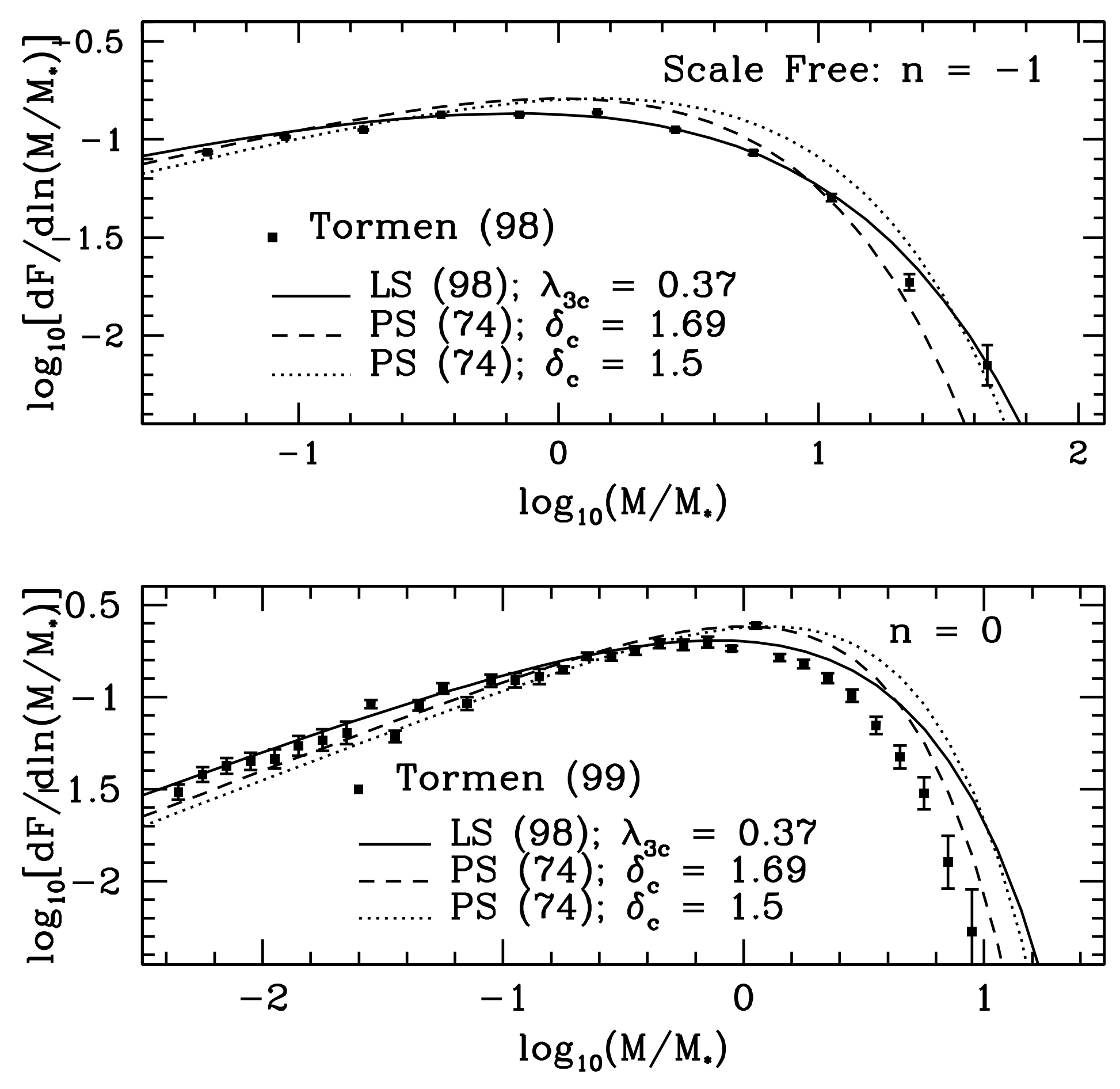


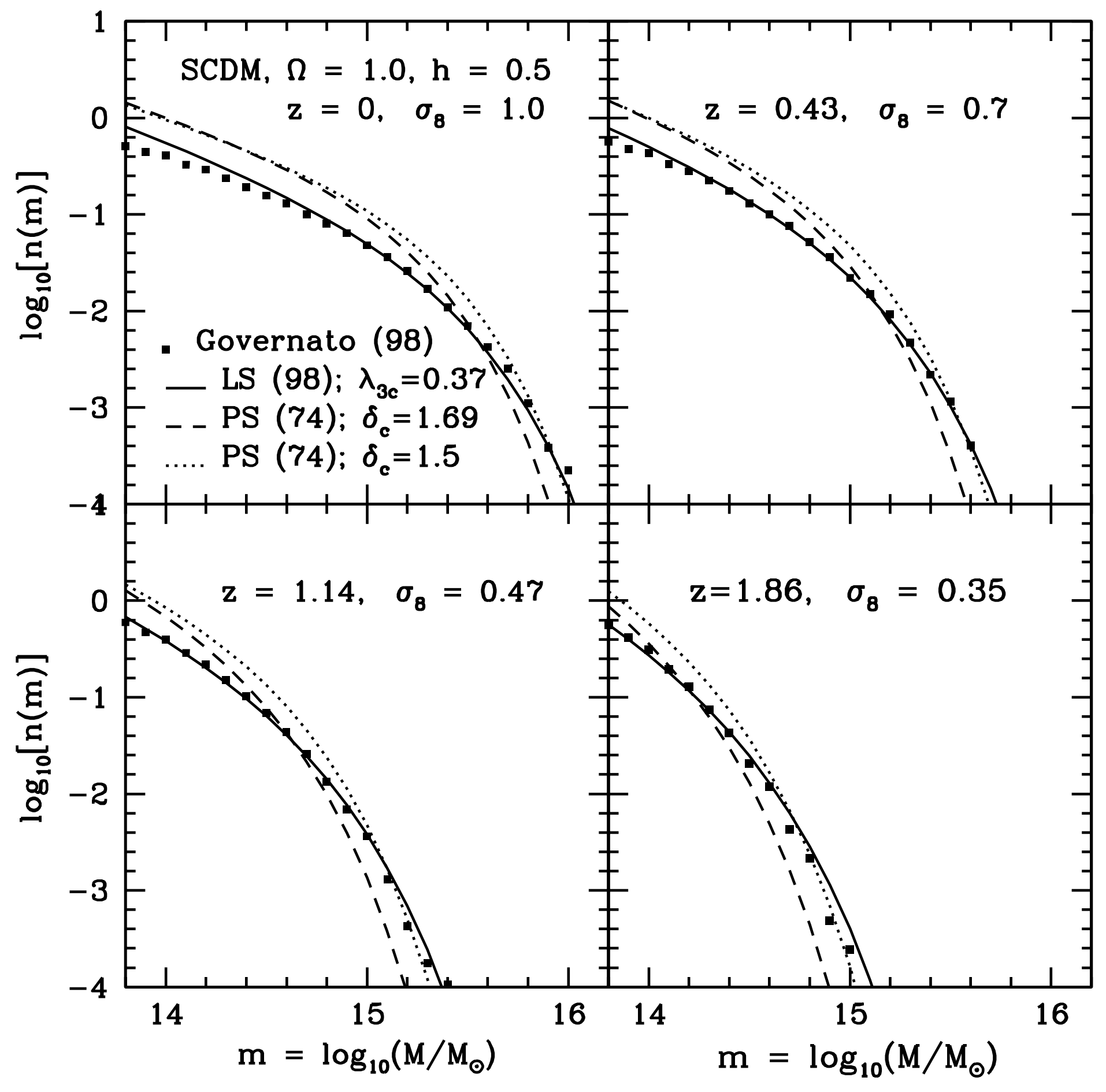



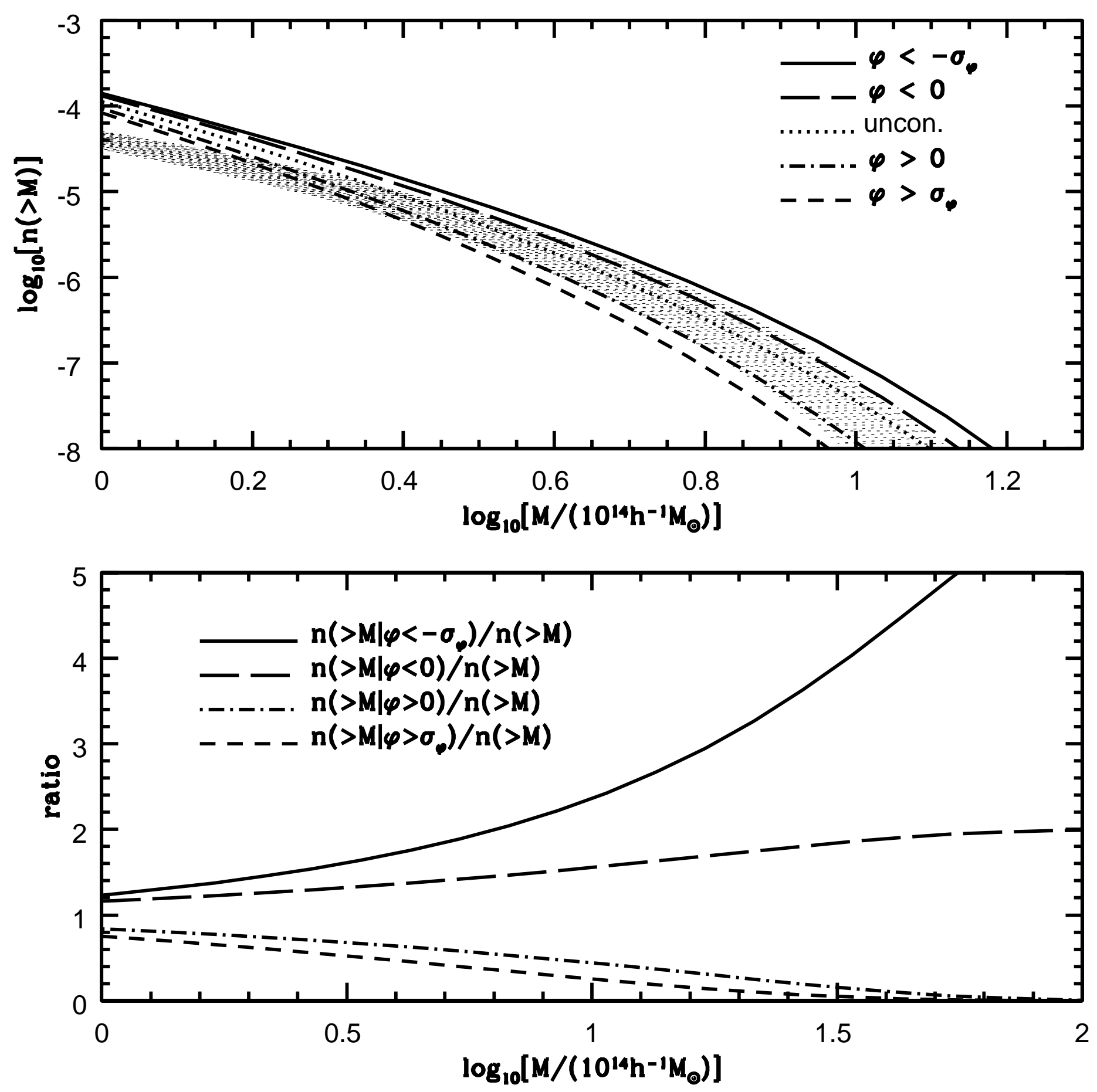


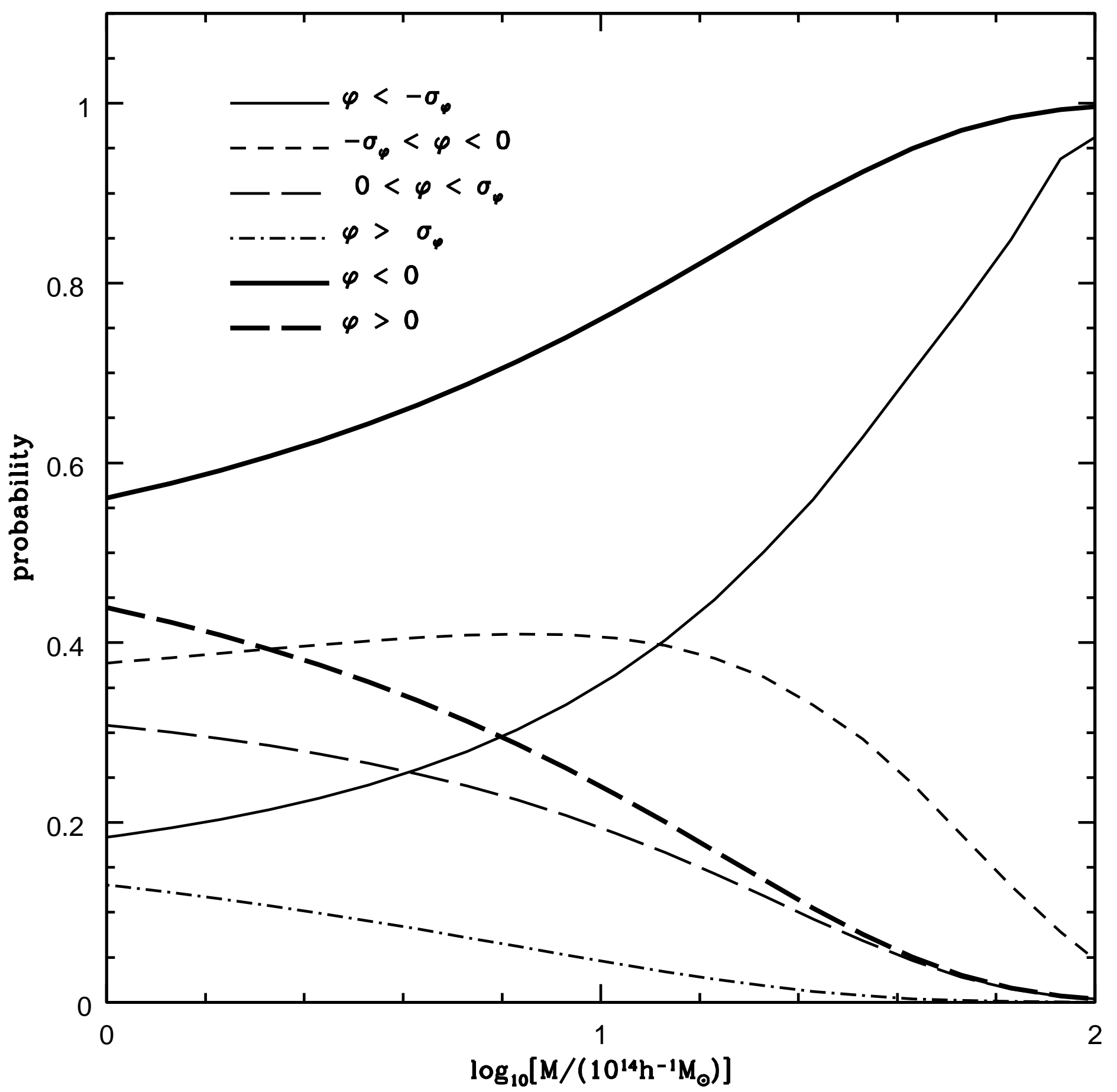

